\title{
Prosthesis Design Based on an Asymmetric Passive Dynamic Walker
}

\author{
John Sushko, Craig Honeycutt, and Kyle B. Reed \\ Department of Mechanical Engineering \\ University of South Florida \\ Tampa, FL, 33620, U.S.A.
}

\begin{abstract}
This paper presents a theoretical design of a transfemoral prosthesis based on physical asymmetries. Breaking the mold of current prostheses, we propose a general prosthetic leg design in which the knee location is shifted off its traditional knee line. The objective is to find an aboveknee prosthetic design for which the overall mass is decreased, especially the below knee portion, while still exhibiting a symmetric gait pattern. A lighter prosthetic that enables a symmetric gait will increase the comfort and decrease the detrimental effects of an asymmetric gait for individuals that wear prostheses. In individuals with prosthetics, changing the knee location could also result in a symmetric gait. To analyze the prosthesis, the existing passive dynamic walker model with five masses was adapted to include nine masses to better represent the mass distribution throughout each limb. A search through the parameter space revealed that a symmetric gait could arise from a system with different knee locations on both sides while the mass of the prosthesis was lighter than the existing leg. In order to accomplish this, the knee location of the prosthesis was positioned below the intact knee by $36.7 \%$ of the total shank length. This physical asymmetry resulted in a below knee mass reduction of $68.2 \%$ and a total mass reduction of $13.4 \%$ while having a symmetric gait. In addition, the model gives further indications on where to add or remove mass to decrease the amount of asymmetry in individuals with prostheses and other causes of asymmetric gait.
\end{abstract}

\section{INTRODUCTION}

This paper extends the mathematical models of passive dynamic walkers (PDWs) to introduce a corporeal example of how they can be used for rehabilitation. This paper also shows how the rotational inertia and center of gravity of each extremity relate to step length patterns. Furthermore, we propose a practical application for this model which suggests a deviation from typical prosthetic designs. Our proposal is a prosthetic design for transfemoral amputees in which the position of the knee is moved to a location dissimilar to the natural leg (see Figure 1). The prosthetic design will allow for certain design benefits such as minimizing mass and volume while still giving the amputee consistent step lengths throughout their gait cycle. The prosthetic knee may be in a slightly different location, which will affect sitting and appearance, much like occurs in knee disarticulation [1]. However, the reduction in mass and benefit of symmetric motion are worthwhile tradeoffs in many instances given the typical discomfort of wearing prosthetic devices.

To validate the possibilities of a prosthesis with physical asymmetries relative to the amputee's non-altered leg, we investigated the effects of moving the rotational inertia without changing the center of mass. A PDW model is used because of its similarity to human gait [11]. Also, because we are investigating purely dynamic effects, a model excluding human cognition is desired. To better explore the effects of

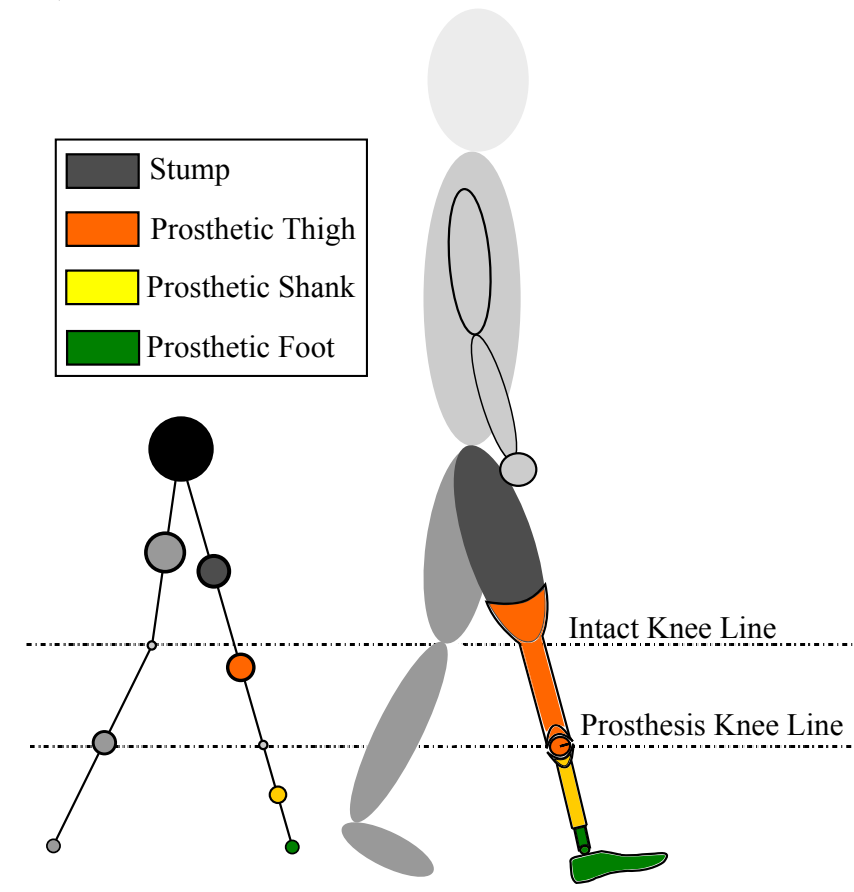

Fig. 1. Left: five mass walker model where thigh and shank masses are in terms of the left and right legs. Right: an exaggerated view of the knee location in a prosthesis that can generate a symmetric gait while reducing the overall mass of the prosthesis.

changing the center of mass and moment of inertia of each leg independently, we created a nine mass model that better describes the distribution of mass throughout the limb. Also, while the old five mass model only accounts for the center of mass of each extremity, the nine mass model can more accurately represent inertia.

\section{BACKGROUND}

Our model is based on a PDW model created by Chen [2]. Derived from the compass gait model, this model divides the gait cycle into four discrete phases. These are: 3-link phase, 2-link phase, knee strike and heel strike. For a successful gait to be established, the energy lost at knee strike and heel strike (the collision events) has to be gained from gravity's effect on the overall inertia of the mechanism. The same energy exchange applies to previous models pioneered by McGeer. In his seminal paper [11], McGeer compared the gait cycle to a rimless wheel with collisions occurring as each spoke made contact with the ground. Expanding on this concept is the compass gait model, which is fundamentally a double pendulum with a hip mass and two leg masses. Chen expanded on this concept by creating a five mass system to more accurately describe a PDW with knees [2]. Our 
previous model, shown in Figure 2, then specified masses in terms of which leg they were associated with, giving us the ability to make physical asymmetries within the model [8].

The effects of modifying physical parameters in a PDW model was accomplished by moving the location of the knee, manipulating the location and magnitude of the masses, and changing the overall length of each leg [8]. In doing this, several different step patterns were found that could relate to gait irregularities in humans. Related research was done by Gregg et al. [6] who changed the ramp angle and initial conditions to get their model to exhibit asymmetric gait patterns. They used a physically symmetric model where changes to the environment evoked asymmetries. In contrast, we tuned our model with physical asymmetries and observed the resulting walking patterns.

This paper further explores the effects of changing masses and mass locations while directly applying these changes to a practical application, prostheses. It has been found that those using a prosthesis exhibit less efficient and sometimes unnatural asymmetric gait patterns [4][7]. This inefficiency is even more evident in transfemoral amputees and because of this, a great deal of effort is put into compensating for these unwanted motions [9]. First, by presenting a nine-mass model, we will demonstrate an asymmetric step pattern and tune it towards symmetry without significantly changing the overall rotational inertia. Then, we will analyze the prosthesis using the nine mass model.

\section{Nine MASS MODEL}

A kneed passive dynamic walker can be modeled as an unactuated multi-pendulum system. The first kneed model was derived by Chen [2]. Chen took the compass gait [5] and added knees and shanks to the system to better describe the human gait. This model has a total of five masses. The legs were differentiated as: the stance leg (st), which is the leg that is in contact with the ground and the swing leg $(s w)$, which is the leg that swings freely. The five mass model can be seen in Figure 2. In [8], one-dimensional tests were performed to evaluate the effects of independently changing the mass of the right shank, the location of the thigh mass, and the location of the knee. This provided us with

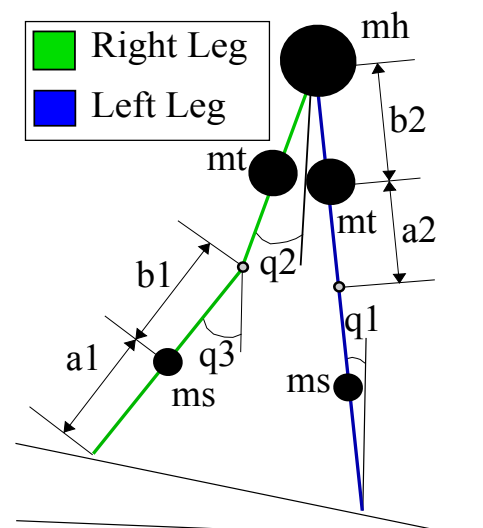

Fig. 2. Five Mass Walker Model: This is also the same model Chen used. For our model $m t$ and $m s$ are in terms of the left and right legs. interesting gait patterns and a basis of how the parameters affect step patterns, but no real steps toward rehabilitation research. This model was then further advanced by adding another mass on each link. Four additional masses were introduced bringing the total to nine. The nine mass system is shown in Figure 3. A major benefit of the nine mass system is that we can better approximate the mass distribution of human legs and the legs of a physical PDW, which will aid in the development of different rehabilitation methods. The nine mass system also gives us a large amount of adjustably and versatility for different model configurations.

The nine masses shown in Figure 3 are the hip mass $(m h)$, the upper shank mass on the stance leg $\left(m s 2_{s t}\right)$, the lower shank mass on the stance leg $\left(m s 1_{s t}\right)$, the upper thigh mass on the stance leg $\left(m t 1_{s t}\right)$, the lower thigh mass on the stance leg $\left(m t 2_{s t}\right)$, the upper shank mass on the swing leg $\left(m s 2_{s w}\right)$, the lower shank mass on the swing leg $\left(m s 1_{s w}\right)$, the upper thigh mass on the swing leg $\left(m t 1_{s w}\right)$, and the lower thigh mass on the swing leg $\left(m t 2_{s w}\right)$. The bottom rods are the shank links and the top rods are the thigh links. The shank length is $l s=a 1+b 1+c 1$ and the thigh length is $l t=a 2+b 2+c 2$, both in terms of $s t$ and $s w$. The total length is $L=l s+l t$, also in terms if $s t$ and $s w$. The walker goes through two distinct stages in its gait pattern: a two-link phase and a three-link phase. The walker starts in the threelink phase where it acts as a three link pendulum system. The three-link phase, shown in Figure 3, is described as $L_{s t}$ which is connected by the hip to $l t_{s w}$ and the knee connects $l t_{s w}$ to $l s_{s w}$. The walker stays in the three-link phase until the knee strike collision occurs. After knee strike, the knee is locked and the system becomes a double pendulum. The double pendulum system is the two-link phase (not shown). The two-link phase has two links $L_{s t}$ and $L_{s w}$ which are connected together by the hip. The walker stays in twolink until the heel strike collision. Heel strike finishes the walker cycle and three-link starts the cycle again. The nine mass system's dynamics are described by the Lagrangian

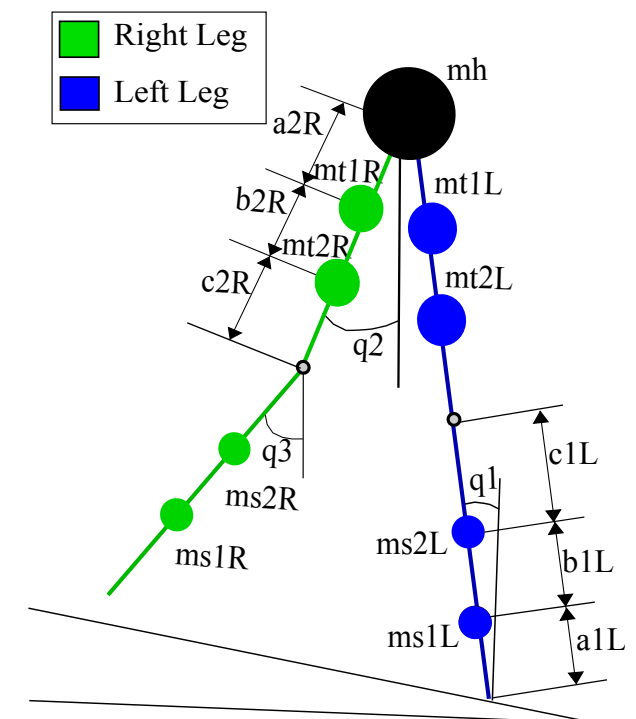

Fig. 3. Nine Mass Three Link Model. Note: $m t 1 m t 2$ and $m s 1 m s 2$ are in terms of the left and right legs. In this figure $c 1 R$. 
formulation for a multi-pendulum system shown in (1),

$$
H(q) \ddot{q}+B(q, \dot{q}) \dot{q}+G(q)=0,
$$

where the three matrices $H, B$, and $G$ are the inertia, velocity, and gravity matrices, respectively. The dynamics for the asymmetric five mass model are derived in [8]. The nine mass model is essentially the five mass model with added terms in the Lagrangian matrices. Equation (2) shows the first term in the inertia matrix for the five mass system,

$$
\begin{aligned}
H_{11}= & m s_{s t} a 1_{s t}^{2}+m t_{s t}\left(l s_{s t}+a 2_{s t}\right)^{2}+ \\
& \left(m h+m s_{s w}+m_{s w}\right) L_{s t}^{2},
\end{aligned}
$$

and (3) is the first term for the nine mass system,

$$
\begin{aligned}
H_{11}= & m s 1_{s t} a 1_{s t}^{2}+m s 2_{s t}\left(a 1_{s t}+b 1_{s t}\right)^{2}+ \\
& m t 2_{s t}\left(l s_{s t}+c 2_{s t}\right)^{2}+m t 1_{s t}\left(l s_{s t}+c 2_{s t}+b 2_{s t}\right)^{2}+ \\
& \left(m h+m s 1_{s w}+m s 2_{s w}+m t 1_{s w}+m t 2_{s w}\right) L_{s t}^{2}
\end{aligned}
$$

As you can see the nine mass equation is quite a bit longer; the full derivations are presented in [14].

\section{A. Nine Mass Model Results}

We chose to derive the nine mass system to make our model more versatile, but do the extra masses make a significant difference? To determine this, we changed the moment of inertia on specific links on the right leg only while keeping the center of mass constant. The two masses move an equal distance away from the baseline location. The parameter that separates the two masses on each link for the right leg was incremented from $0 m$ to $0.5 m$ (i.e., $b 1 R$ and $b 2 R$ ). There were three tests performed by incrementing $b 1 R$ and $b 2 R$ separately and both $b 1 R$ and $b 2 R$ simultaneously. We were trying to find the value of these parameters that produced the largest asymmetry but still was able to walk successfully and stably. Step length difference is used since it is a common description of asymmetry in human gait studies [12]. For a test to be successful the walker had to walk for 50 steps. The largest asymmetry for the shank is 0.0212 , which occurs when the two masses are separated by $b 1 R=0.24 m$, which is shown in Figure 4a. When the two thigh masses are separated by a distance $b 2 R=0.25$, the system produces a step length difference of $0.0297 \mathrm{~m}$, shown in Figure $4 \mathrm{~b}$. The combined thigh and shank test produced an asymmetry of $0.029 \mathrm{~m}$ when the shank masses are separated by $b 1 R=0.03 \mathrm{~m}$ and the thigh masses by $b 2 R=0.26 \mathrm{~m}$, shown in Figure 4c. These results show that the moment of inertia alone can substantially influence the gait pattern. Note that all of these asymmetries arise from changing the moment of inertia on the right leg only while keeping the left leg parameters held constant.

Next, the configurations with the largest asymmetries arising from changing the right leg only were used as a baseline to test the effect of shifting the center of mass location on the left leg shank and thigh. In other words, the center of mass was changed on the leg opposite from the leg that the moment of inertia was changed on. This test will determine if the asymmetry can be eliminated by changing the center of mass on the left leg. The two masses on the

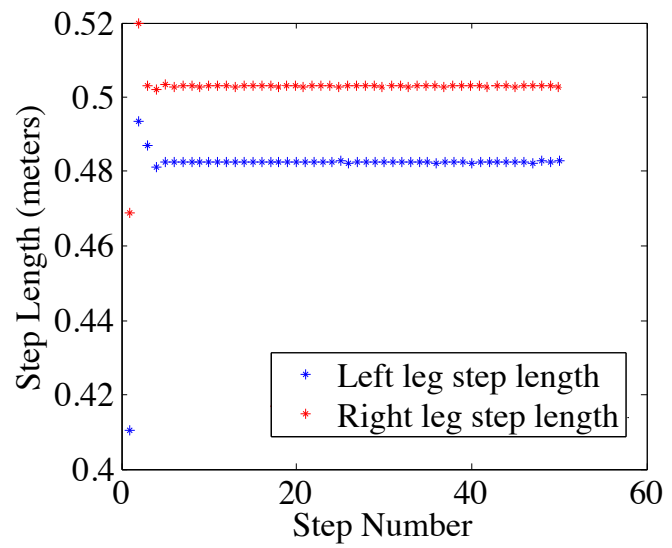

(a) This step length plot shows the asymmetry that arises when the separation distance between the two shank masses is $0.24 \mathrm{~m}$.

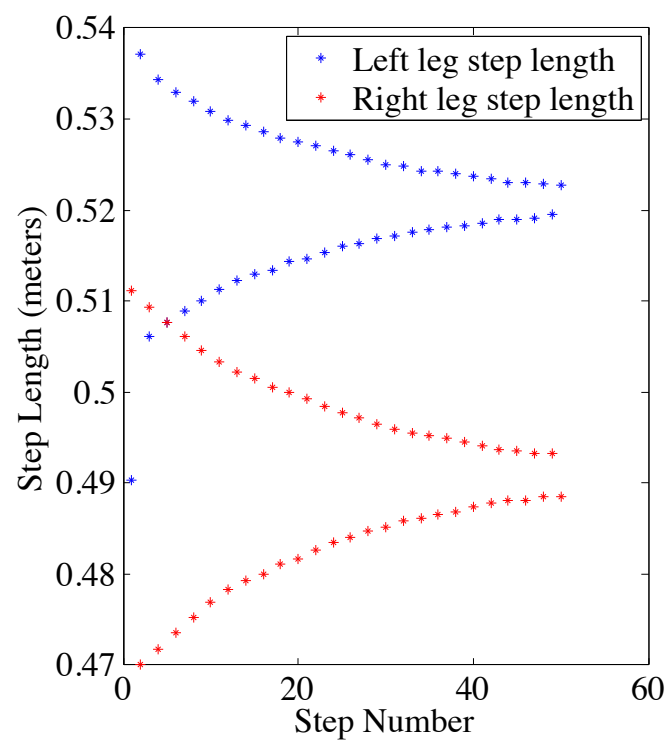

(b) This step length plot shows the asymmetry that arises when the separation distance between the two thigh masses is $0.25 \mathrm{~m}$.

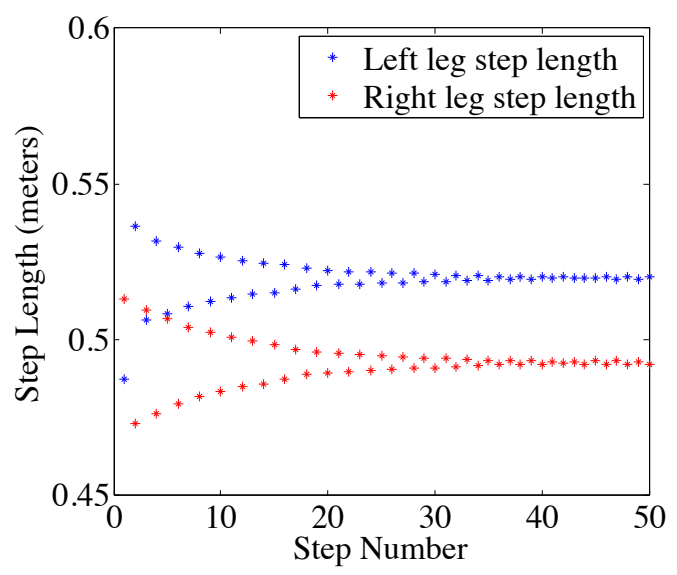

(c) This step length plot shows the asymmetry when the two masses on the shank are separated by $0.03 \mathrm{~m}$ and the two thigh masses are separated by $0.26 \mathrm{~m}$.

Fig. 4. These step length plots show that an asymmetry arises due to the altered moment of inertia when the two masses on the right leg thigh and/or shank are separated. Note that it can take several steps (over 50 in (b)) to establish a constant pattern. 


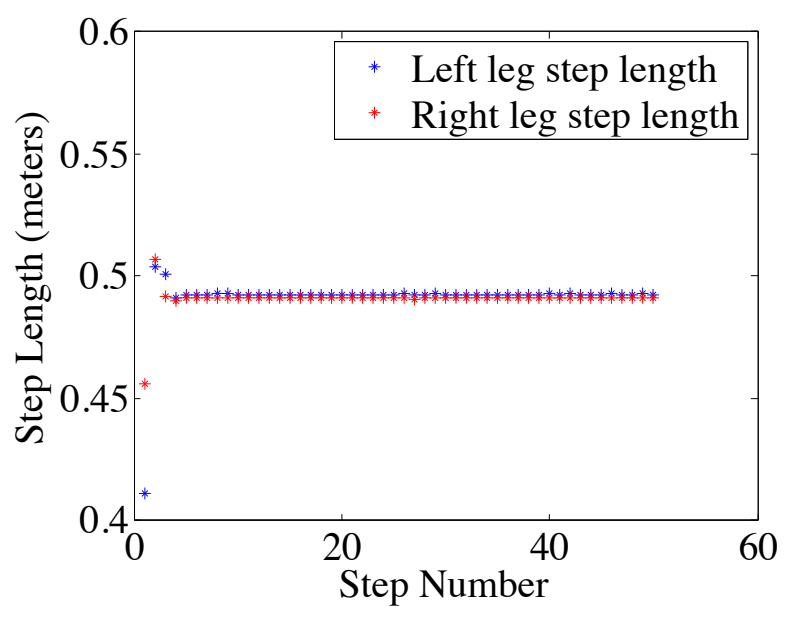

(a) This shows the symmetric solution when the right shank masses are separated $(b 1 R=0.24 \mathrm{~m})$ and $c 1 L=a 2 L=0.24 \mathrm{~m}$.

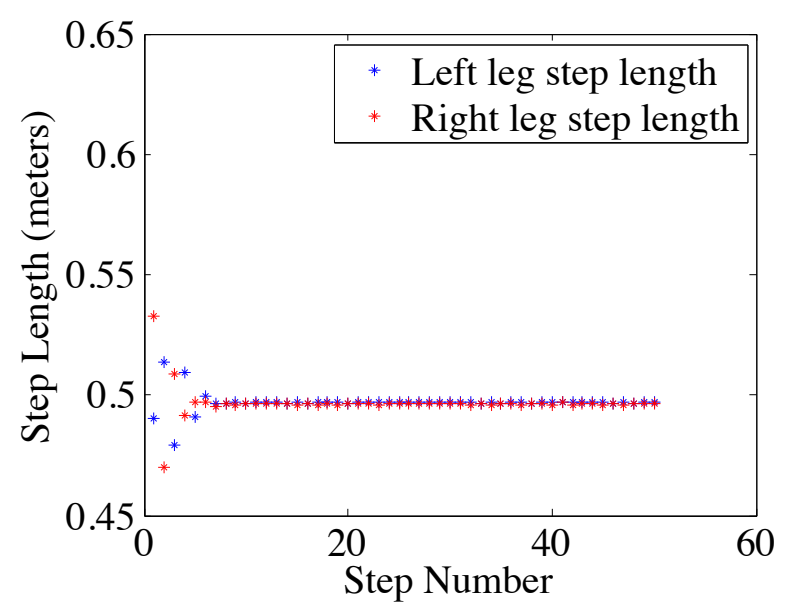

(b) This shows the symmetric solution when the right thigh masses are separated $(b 2 R=0.25 \mathrm{~m})$ and $c 1 L=0.17$ and $a 2 L=0.25$.

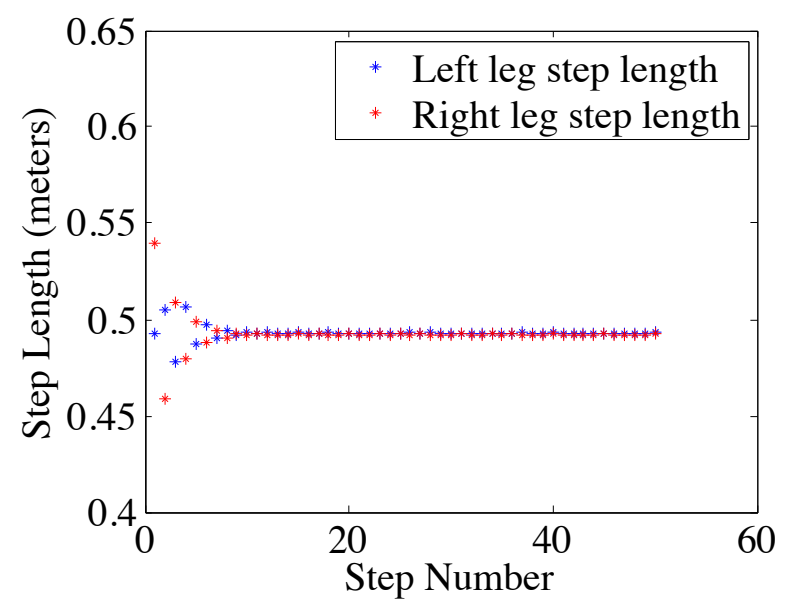

(c) This shows the symmetric solution when the masses are separated on both the shank $(b 1 R=0.03)$ and the thigh $(b 2 R=0.26 m)$ and $c 1 L=0.14 m$ and $a 2 L=0.23 m$.

Fig. 5. These plots show the symmetric gaits generated by altering only the left leg of the asymmetric models generated in Figure 4 that were made asymmetric by changing only the right leg. Each subfigure in Figure 4 corresponds to those shown here (e.g., $4 a \rightarrow 5 a$ ). left leg segments were located at the same location by setting $b 1 L$ and $b 2 L$ to zero. Essentially we made two large masses, one on the shank and one on the thigh. Note that the center of mass and the moment of inertia of the left leg are coupled while the location of the one larger mass is moved. This is unlike the right leg where the center of mass stays the same and the moment of inertia varies.

The values of $c 1 L$ and $a 2 L$ were independently iterated from $0 m$ to $0.5 m$ with all permutations evaluated by a brute force search. The values of $a 1 L$ and $c 2 L$ were modified in each case to maintain the same total shank and total thigh lengths. There are certain parameters that lead to a symmetric gait pattern when the center of mass is moved along both the shank and the thigh. In Figure 5 it can be seen that the walker can return to symmetry when the masses on the left leg are at a specific location. These are the step length plots which plot the step length vs. the step number. Figure 5a shows the symmetric solution to the asymmetry created in Figure 4a which is the asymmetry that arises when the masses on the right shank are spread out by $0.24 \mathrm{~m}$. This created asymmetry is canceled out when the large coupled masses on the left leg shank and thigh are both moved to $0.01 \mathrm{~m}$ above the center of the shank and think links, respectively. Figure $5 \mathrm{~b}$ shows the diminished asymmetry initially caused by the right leg thigh masses being separated by $0.25 \mathrm{~m}$. The reduction happens when the coupled masses on the left leg shank are moved to $0.17 \mathrm{~m}$ below the knee and the thigh mass is in the center of the thigh link (i.e., $a 2 L=0.25$ ). Figure $5 \mathrm{c}$ shows that the asymmetry in Figure 4c was eliminated when the shank and thigh mass were moved to $0.14 \mathrm{~m}$ below the knee and $0.23 \mathrm{~m}$ below the hip. This shows that with a specified center of mass and moment of inertia for one leg, the walker can be tuned to symmetry by holding the center of mass constant and varying the moment of inertia of the opposite leg.

We are also interested in how the step length difference changes as the moment of inertia changes. Using the data from the test when $b 2 R$ is $0.25 m$, we iterated $c 1 L$ and $a 2 L$ each from $0 \mathrm{~m}$ to $0.5 \mathrm{~m}$. We then plotted the moment of inertia for each leg vs. the step length difference. This trend is shown in Figure 6a. The changing moment of inertia of the left leg is represented by the black dots, and the constant moment of inertia on the right leg is represented by the solid red line. As shown in Figure 6a, the moment of inertia of the left leg is dependent upon the location of the mass. The location of the shank and thigh masses on the left leg changes with $c 1 L$ and $a 2 L$, which is depicted by the colored lines in Figure 6a. When $c 1 L$ is approximately $0.3 m$, the walker will be more stable. However, if $a 2 L$ is too large the step length difference will increase making the walker more unstable. This can also be seen in Figure 6b, which is a three dimensional plot of $a 1 L$ vs. $c 2 L$ vs. step length difference. It shows that there are a range of values of $c 1 L$ and $a 2 L$ that produce a symmetric walker given an altered moment of inertia on the opposite leg. Because of this, there would be many solutions to an optimization problem based solely on step symmetry, thus the following results used a brute force solution and we manually selected several specific configurations to highlight. 


\section{Prosthetic Model}

For the prosthesis tests, we modified our nine mass model based on anthropomorphic data [3], which gave the location and magnitude of the center of mass for each part of the leg. We took these values and allowed them to vary until we generated a stable and symmetric gait pattern. The values of the parameters for the anthropomorphic model are listed in Tables I and II. Then, to develop a model for the prosthesis, we assumed an amputation point of two thirds of the total thigh length distally from the hip. We then iterated through different knee locations and masses of the prosthetic thigh and shank until we found step length symmetry in the gait.

Figure 1 shows the mass distribution of both the intact and prosthetic legs. For the intact leg, we placed both thigh

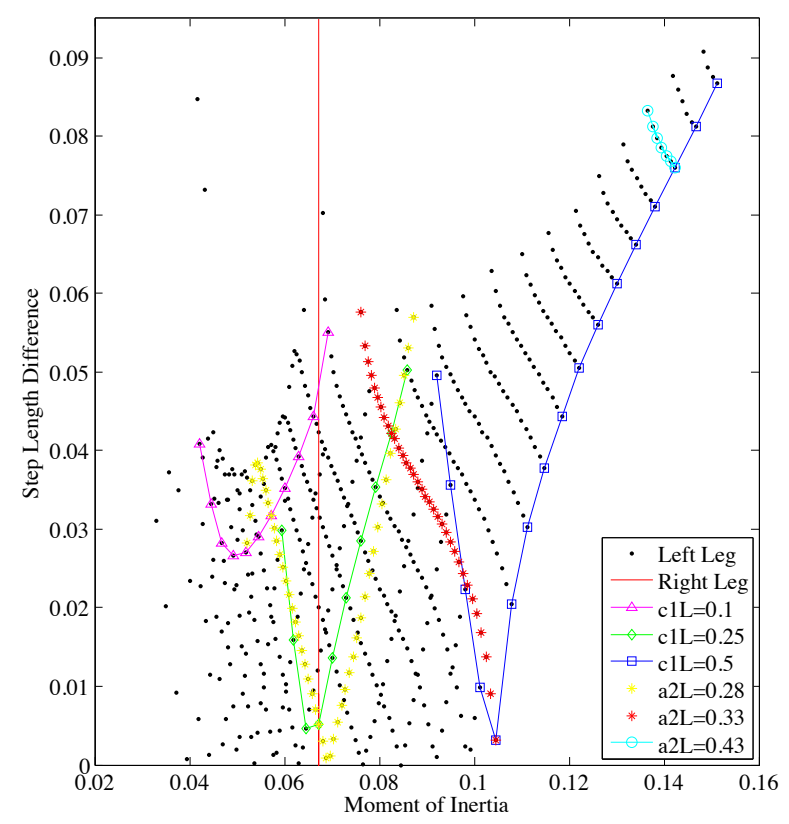

(a) Moment of inertia of the left leg when the right leg thigh mass is spread out by $0.25 \mathrm{~m}$.

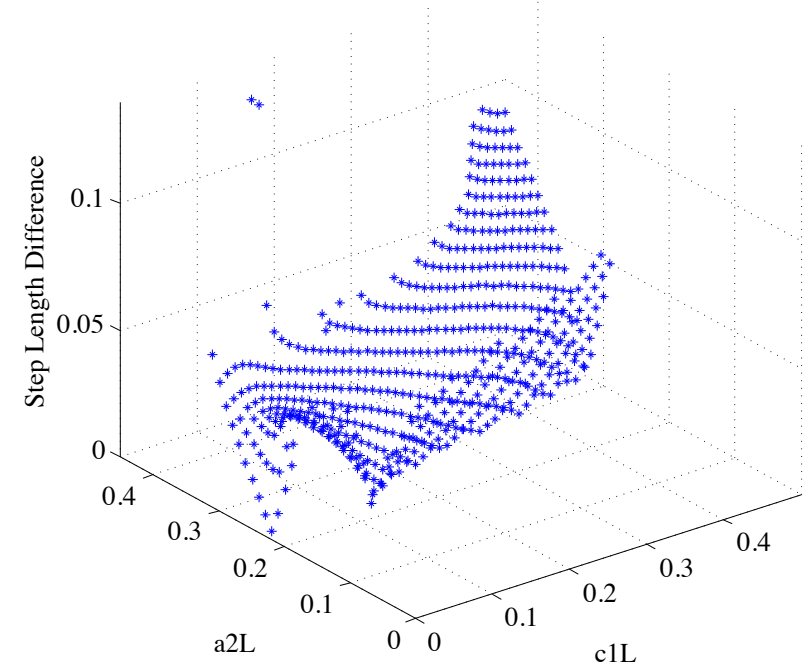

(b) a21 and c1L vs. Step Length Difference

Fig. 6. The success and failure of the different parameters and the step lengths of each leg over all the parameters tested. masses at the center of mass given by the anthropomorphic model and gave them each a value one half of the total thigh mass, simulating a single mass. We used one shank mass for the center of mass of the shank and moved the other down to represent the foot's center of mass. A similar distribution was used for the prosthetic leg with the exception of the thigh masses. Here, one thigh mass was used to represent the center of mass of the stump while the other was used for the center of mass of the prosthetic thigh. In Figure 1, you can equate the point masses on the model with the colored sections of the prosthetic model. From top to bottom: Dark grey represents the mass of the stump, orange represents the prosthetic thigh, yellow equates to the mass of the prosthetic shank, and green represents the foot mass. Also, notice the difference between the prosthesis and natural knee line.

\section{A. Prosthetic Model Results}

This prosthetic model is used in our research to break the assumption that the transfemoral prosthetic knee location should be the same as the existing knee. Unlike existing prostheses, tuning the parameters of our prosthetic model could lead to more symmetric gait patterns but reduce energy cost. Mattes et al. note that mass of the prosthetic limb and energy costs are proportional [10]. Thus, we are trying to reduce the mass of the prosthesis while moving the knee location to keep a symmetric gait pattern. In addition, this model can be used to give insights on how to reduce the asymmetry in individuals that wear existing prostheses.

To find a lighter, prosthesis we set up a brute force search. This program iterated through a range of values for the prosthetic thigh, the prosthetic shank, and the knee location. The knee location is moved by changing $c 1 R$ and $c 2 R$ in relation to each other. The ranges for these values are shown in Table III. All permutations and combinations were evaluated. Then, post processing analyzed the data to find combinations that were optimal. The most ideal or optimal combination is when the mass of the prosthetic limb is reduced significantly but the gait is still stable.

Although there are many solutions, we will discuss the three prosthesis configurations, compared in Table IV. As a baseline, the first configuration is when the knee location is in the same location as the knee of the intact leg. For

TABLE I

ANTHROPOMORPHIC MODEL MASS

\begin{tabular}{lllll}
\hline \hline & $\begin{array}{l}\text { Mass of } \\
\text { hip }(\mathrm{kg})\end{array}$ & $\begin{array}{l}\text { Mass of } \\
\text { thigh }(\mathrm{kg})\end{array}$ & $\begin{array}{l}\text { Mass of } \\
\text { shank }(\mathrm{kg})\end{array}$ & $\begin{array}{l}\text { Mass of } \\
\text { foot }(\mathrm{kg})\end{array}$ \\
\hline Given Masses & 0.532 & 0.115 & 0.044 & 0.019 \\
Derived Model & 0.532 & 0.315 & 0.100 & 0.019 \\
\hline
\end{tabular}

TABLE II

\begin{tabular}{llll}
\multicolumn{4}{c}{ ANTHROPOMORPHIC MODEL MASS LOCATIONS } \\
\hline \hline $\begin{array}{l}\text { Walker } \\
\text { parameter }\end{array}$ & $\begin{array}{l}\text { Center of mass } \\
\text { location from } \\
\text { proximal endpoint }\end{array}$ & $\begin{array}{l}\text { Calculated } \\
\text { length }(\mathrm{m})\end{array}$ & $\begin{array}{l}\text { Model } \\
\text { location }\end{array}$ \\
\hline Hip & 0.540 & 0.000 & Top of walker \\
Thigh & 0.433 & 0.217 & From hip \\
Knee & 0.000 & 0.239 & From thigh \\
Shank & 0.433 & 0.217 & From knee \\
Foot & 0.429 & 0.239 & From shank \\
\hline
\end{tabular}


TABLE III

PRosthesis DATA RANGES

\begin{tabular}{lccc}
\hline \hline Parameter & Low Value & High Value & \# increments \\
\hline Prosthetic Thigh & 0 & 0.1575 & 16 \\
Prosthetic Shank & 0 & 0.10 & 16 \\
Knee Location (c1R) & 0.0057 & 0.25 & 16 \\
\hline
\end{tabular}

TABLE IV

Prosthesis Model Mass Results

\begin{tabular}{llll}
\hline \hline Configuration & Thigh Mass & Shank Mass & Total Mass \\
\hline $\begin{array}{l}\text { Heavier } \\
\text { Symmetric }\end{array}$ & $17 \%$ increase & $38 \%$ decrease & $2 \%$ increase \\
$\begin{array}{l}\text { Lighter } \\
\text { Symmetric }\end{array}$ & $7.3 \%$ increase & $68 \%$ decrease & $13.4 \%$ decrease \\
$\begin{array}{l}\text { Lighter } \\
\text { Asymmetric }\end{array}$ & $2 \%$ decrease & $63 \%$ decrease & $19 \%$ decrease \\
\hline
\end{tabular}

this configuration to walk symmetrically the total mass of the prosthetic leg had to be increased by $2 \%$. For this $2 \%$ leg mass increase, the thigh mass increased by $17 \%$ while the shank mass decreased by $38 \%$. The second configuration achieved a lighter symmetric prosthesis by moving the knee mass down by $36.7 \%$ of the total shank length. In doing this, we were able to reduce the shank mass by $68 \%$ with a thigh mass increase of $7.3 \%$. This resulted in a $13.4 \%$ reduction of the total mass of the prosthetic leg. Even though this is a small total mass reduction, the important reduction is in the shank which was fairly dramatic.

Individuals that wear a prostheses typically have an asymmetry where their prosthetic leg has a step length that is longer than their intact leg [10]. Our first two configurations have shown that we can minimize this asymmetry, but the third configuration demonstrates that we can go past the symmetric point and have the prosthetic leg produce a shorter step length than the intact leg. This configuration would imply that the prosthetic leg is overcompensating for the asymmetry that the wearer is likely to develop. To get this step length outcome, the knee was moved down by $42.8 \%$, which caused the prosthetic shank mass to be reduced by $63 \%$ and the prosthetic thigh mass to be reduced by $2 \%$. This reduced the entire prosthetic leg mass by $19 \%$. By moving the knee down and reducing the masses, the step length of the prosthesis was $7.4 \%$ less than the step length of the intact leg. This outcome shows we can theoretically tune a prosthesis to be lighter than the intact leg while overcompensating for the wearer's developed asymmetry.

\section{CONClusions And Future Work}

This research is the expansion of previous passive dynamic walker models to a nine mass model, which allows for more adjustability, versatility, and better approximates the human leg mass distribution. We have shown that the nine mass model has improvements over the traditional five mass model, which forces the center of mass to change with the moment of inertia. The nine mass model allows the moment of inertia to be changed while keeping the center of mass constant and vise versa. The ability to adjust the mass locations to any specific walker configuration is very useful when describing an anthropomorphic model. We were able to create a theoretical transfemoral prosthetic that was lighter than the intact leg when the knee location was moved down. We were able to achieve a $68 \%$ decrease in the prosthetic shank mass and a $13.4 \%$ decrease in the total prosthetic mass while maintaining a symmetric gait pattern. This goes against traditional prosthetic designs that put the prosthetic knee in the same location as the intact knee.

An important aspect of this work is human validation. Preliminary tests have demonstrated that changing simple parameters such as adding a mass on one foot or changing the height of a shoe will lead to gait changes as predicted by the model. However, these comparisons need to be with individuals who wear prostheses.

This model of walking can be extended to analyze how the joint torques affect the stress on the joints. As opposed to simply looking for symmetric steps, which is one important parameter for efficient gait, analyzing the stress on joints could be another measure that is minimized.

Another extension of this model is to look at how spasticity, the tightness in the muscles arising from a stroke [13], affects gait patterns. The model of joint spasticity can be modeled by using velocity dependent damping and position dependent stiffness on one of the knees in the PDW model. Then, like done in this paper, the developed asymmetry that arises can be reduced by changing other parameters in the model.

\section{REFERENCES}

[1] RF Baumgartner. Knee disarticulation versus above-knee amputation. Prosthetics and Orthotics International, 3(1):15-19, 1979.

[2] Vanessa F. Hsu Chen. Passive Dynamic Walking with Knees: A Point Foot Model. Master's thesis, MIT, 2005.

[3] R. Drillis et al. Body segment parameters: a survey of measurement techniques. National Academy of Sciences, 1964.

[4] Andrew Gitter, Joseph Czerniecki, and Kelly Weaver. A reassessment of center-of-mass dynamics as a determinate of the metabolic inefficiency of above-knee amputee ambulation. American Journal of Physical Medicine and Rehabilitation, 74(5), 1995.

[5] Ambarish Goswami, Benoit Thuilot, and Bernard Espiau. A Study of the Passive Gait of a Compass-Like Biped Robot. The International Journal of Robotics Research, 17(12):1282-1301, 1998.

[6] R. D. Gregg, A. Degani, Y. Dhahe, and K. M. Lynch. The basic mechanics of bipedal walking lead to asymmetric behavior. In Proc. IEEE Int. Conf. Rehabilitation Robotics, pages 816-821, 2011.

[7] Martin D. Hoffman, Lois M. Sheldahl, Kenneth J. Buley, and Paul R. Sandford. Physiological comparison of walking among bilateral aboveknee amputee and able-bodied subjects, and a model to account for the differences in metabolic cost,. Archives of Physical Medicine and Rehabilitation, 78(4):385 - 392, 1997.

[8] C. Honeycutt, J. Sushko, and K. B. Reed. Asymmetric passive dynamic walker. In Proc. IEEE Int. Conf. Rehabilitation Robotics, 2011.

[9] C.T. Huang, J. R. Jackson, N. B. Moore, P. R. Fine, K. V. Kuhlemeier, G. H. Traugh, and Saunders P. T. Amputation: energy cost of ambulation. Arch Phys Med Rehabil, 60(1):18-24, 1979.

[10] S. J. Mattes, P. E. M., and T. D. Royer. Walking symmetry and energy cost in persons with unilateral transtibial amputations: Matching prosthetic and intact limb inertial properties. Archives of Physical Medicine and Rehabilitation, 81(5):561 - 568, 2000.

[11] Tad McGeer. Passive Dynamic Walking. The International Journal of Robotics Research, 9(2):62-82, 1990.

[12] D. Reisman, H. Block, and A. Bastian. Interlimb Coordination During Locomotion: What Can be Adapted and Stored? J. Neurophysiol, 94(4):2403-2415, 2005.

[13] T. D. Sanger, M. R. Delgado, D. Gaebler-Spira, M. Hallett, and J. W. Mink. Classification and definition of disorders causing hypertonia in childhood. Pediatrics, 111(1):e89-97, 2003.

[14] J. Sushko. Asymmetric passive dynamic walker used to examine gai rehabilitation methods. Master's thesis, Univ. of South Florida, 2011. 